\title{
Validación de un cuestionario breve para detectar situaciones de violencia de género en las consultas clínicas
}

\author{
María Pía Majdalani ${ }^{1}$ María Alemán, ${ }^{1}$ Roberto Fayanás, ${ }^{1}$ \\ Alexandra Guedes ${ }^{2}$ y Raúl Mariano Mejía ${ }^{1}$
}

Forma de citar Majdalani MP, Alemán M, Fayanás R, Guedes A, Mejía RM. Validación de un cuestionario breve para detectar situaciones de violencia de género en las consultas clínicas. Rev Panam Salud Publica. 2005; 17(2):79-83.

RESUMEN Objetivos. Validar un cuestionario diseñado para detectar casos de violencia, en consultorios de atención primaria, hacia mujeres de habla hispana.

Métodos. El estudio de validación se llevó a cabo entre octubre de 2002 y octubre de 2003 en el Programa de Medicina Interna General del Hospital de Clínicas, Universidad de Buenos Aires, Argentina. El cuestionario original en español se había formulado en clínicas de salud sexual y reproductiva de cinco países de América Latina y el Caribe. En él se abarcaban las formas más frecuentes de violencia basada en el género (VBG) (emocional, física, sexual y abuso sexual durante la niñez); se exploraba cómo, cuándo y quién perpetró la agresión, y se indagaba acerca de la percepción de la paciente con respecto al peligro en que se encontraba. Durante la investigación en Buenos Aires se evaluaron el formato y el contenido teórico de la encuesta y se realizaron validaciones lingüística y psicométrica. Se evaluó la confiabilidad del instrumento en términos de reproducibilidad, estabilidad y coherencia interna.

Resultados. Se comprobó que las mujeres encuestadas comprendieron bien las preguntas, los ejemplos dados y las opciones de respuesta y que no percibieron la encuesta como un elemento de intimidación, ni una intromisión en su vida privada. En general consideraron su aplicación en el ámbito de la atención primaria como una oportunidad clave para expresar su sufrimiento y recibir ayuda. El nivel de concordancia entre las respuestas en las cinco áreas exploradas (violencia psicológica, violencia psíquica, violencia sexual, abuso sexual en la niñez y percepción del riesgo actual) fue muy alta, tanto en lo que respecta a reproducibilidad (valores de kappa de 0,63 a 1,00) como a estabilidad (valores de kappa de 0,62 a 1,00). La coherencia interna, evaluada mediante el coeficiente de correlación alfa, fue de 0,755 entre la violencia psíquica y la física, de 0,498 entre la violencia psíquica y la sexual, de 0,596 entre la violencia física y la sexual, y de 0,715 entre las diferentes manifestaciones de violencia (psíquica, física, sexual y abuso sexual durante la niñez).

Conclusión. Se demostró la solidez de este cuestionario breve de evaluación, tanto por su formato y su contenido como por su carácter integral y confiabilidad. Este cuestionario puede emplearse en las consultas clínicas para detectar situaciones de violencia psíquica, física y sexual, así como de abuso sexual durante la niñez.

Palabras clave Violencia doméstica, estudios de validación, Argentina.

1 Programa de Medicina Interna General del Hospital de Clínicas, Universidad de Buenos Aires, Argentina. La correspondencia debe dirigirse a: María Pía Majdalani, Programa de Medicina Interna General, Hospital de Clínicas, Universidad de Buenos Aires, Conesa 756 piso 7, Dpto. A (1426) CABA Ar- gentina. Correo electrónico: piamajdalani@yahoo. com.ar

2 Federación Internacional de Planificación Familiar, Región Hemisferio Occidental (IPPF-RHO), Nueva York, Estados Unidos de América.
En 1996, la 49. a Asamblea Mundial de la Salud declaró que la violencia era uno de los principales problemas de salud pública en el mundo (1). En par- 
ticular, la violencia hacia la mujer es en la actualidad una acuciante preocupación para las autoridades nacionales y los organismos internacionales vinculados con la salud $(2,3)$. Abordar la violencia basada en el género (VBG) es algo muy complejo debido a los muchos elementos socioculturales que inciden en ella, entre los cuales se destaca el hecho de que estas situaciones se perciben aún como un problema de índole privada.

Según datos poblacionales recientes de distintas regiones, aproximadamente 20 a 50\% de las mujeres son víctimas de actos de violencia física perpetrados por su pareja actual o previa. Diversas investigaciones apuntan a que la violencia física casi siempre se acompaña de actos de violencia psíquica y que entre un tercio y la mitad de los casos sufren también de violencia sexual. La prevalencia de la violencia hacia la mujer durante el embarazo (de 4 a $8 \%$ ) es mayor que la de preeclampsia y la de la diabetes gestacional (4). Se estima que $40 \%$ de la población femenina de la Argentina sufre maltratos físico y psíquico por parte de su pareja, independientemente de su nivel socioeconómico (5).

La VBG tiene una gran repercusión sobre la salud psíquica y física de la mujer y se ha demostrado que a corto, mediano y largo plazos genera síntomas que son motivos frecuentes de consulta en los servicios de atención primaria de salud (5-8).

Se calcula que la prevalencia de víctimas de VBG en la población atendida en las instituciones del sistema de salud es mayor que en la población en general. El médico de atención primaria se encuentra en una posición clave para detectar estas situaciones, ya que la consulta médica podría ser la única oportunidad que tienen muchas mujeres para hablar de estos hechos con una persona ajena al entorno familiar.

Para la detección y caracterización de los casos de VBG existen numerosos instrumentos en idioma inglés, como el propuesto por Mc Farlane (9), la escala de tácticas de conflictos (10), una escala para mujeres golpeadas (8) y un índice para evaluar el abuso conyugal (11).

Para la detección y caracterización de los casos de VBG entre mujeres lati- noamericanas, la Federación Internacional de Planificación de la Familia, Región Hemisferio Occidental (IPPF$\mathrm{RHO})$, formuló una encuesta dirigida a establecer la detección sistemática de casos de VBG en los centros asistenciales de salud sexual y reproductiva (12). Durante el proceso de validación inicial, realizado en Venezuela, se constató que el número de casos de VBG detectados mediante esta herramienta fue mucho mayor que el logrado mediante el método tradicional basado en preguntas menos específicas y sistemáticas $(40 \%$ frente a $8 \%$, respectivamente). Además, las entrevistadas lograron calificar el tipo de violencia e identificar al agresor (12).

El objetivo de esta investigación fue validar un cuestionario diseñado para detectar casos de violencia ejercidos sobre mujeres de habla hispana que acuden a consultas de atención primaria.

\section{MATERIALES Y MÉTODOS}

El estudio de validación se llevó a cabo entre octubre de 2002 y octubre de 2003 en el Programa de Medicina Interna General (PMIG) del Hospital de Clínicas, Universidad de Buenos Aires, Argentina. El cuestionario empleado se desarrolló originalmente en español en clínicas de salud sexual y reproductiva de cinco países de América Latina y el Caribe, con el apoyo del IPPF-RHO. El cuestionario inicial constaba de cuatro preguntas correspondientes a las definiciones operativas de las formas de VBG más frecuentes (emocional, física, sexual y abuso sexual durante la niñez) y que exploraban cómo y cuándo ocurrió el hecho y quién perpetró la agresión; así como una pregunta sobre la situación actual de riesgo en que se encontraba la paciente. El proceso de validación se realizó en dos etapas.

\section{Primera etapa}

Como primer paso se presentó el proyecto de validación a un representante de los autores de la versión original del cuestionario, quien dio su autorización para utilizarla en este es- tudio. Se realizaron cuatro reuniones semanales entre profesionales del Equipo de Trabajo en VBG y médicos clínicos del PMIG, en las que se evaluaron tanto el formato de la encuesta como su contenido teórico, con el fin de garantizar que cada pregunta expresara la definición conceptual de las manifestaciones de violencia que se debían explorar. Se llegó a un consenso acerca de la redacción de los enunciados de las preguntas, los ejemplos de agresiones y las opciones de respuesta acerca de quién cometió los actos de violencia, las etapas de la vida en que sucedieron y si ocurrían en la actualidad.

Para la validación lingüística del cuestionario se realizaron entrevistas semiestructuradas y en profundidad que fueron grabadas y transcritas en papel para su análisis cualitativo. Se entrevistó a doce mujeres de 23 a 59 años de edad, ocho de ellas con menos de 8 años de escolaridad. Las entrevistó a se realizaron según una guía de pautas (cuadro 1), de modo que se pudieran evaluar la comprensión, la interpretación, el léxico, la reformulación, la aceptación y la pertinencia de los contenidos, tanto para la formulación de los enunciados como para los ejemplos y las opciones de respuesta de cada pregunta.

\section{Segunda etapa}

Una vez validado el cuestionario desde el punto de vista lingüístico, se procedió a su validación psicométrica. Para ello, se conformaron dos grupos a partir de una serie consecutiva de mujeres mayores de 18 años que concurrían a la consulta clínica y que aceptaron participar voluntariamente en esta investigación. Las 32 mujeres del grupo denominado $\mathrm{AB}$ fueron entrevistadas por dos profesionales diferentes y las 35 mujeres del grupo denominado CD fueron entrevistadas dos veces por un mismo profesional con un intervalo de 7-10 días. La asignación a uno $u$ otro grupo dependió de la factibilidad de realizar la segunda entrevista y se realizó de manera tal que ambos grupos tuvieran el mismo número de mujeres.

La confiabilidad del cuestionario se evaluó mediante su reproducibilidad (la 
CUADRO 1. Pautas para las entrevistas en profundidad realizadas durante la validación lingüística del cuestionario de detección de casos de violencia basada en el género

Para el enunciado

1) Leer detenidamente el enunciado de la pregunta (la entrevistadora)

2) ¿Hay alguna palabra de la cual no conoce el significado?

3) ¿Entiende la pregunta?

4) ¿Encuentra alguna dificultad para comprender la pregunta?

5) Explique con sus palabras qué quiere preguntar esta pregunta. Ejemplos.

6) ¿Cómo haría usted esta pregunta? Ejemplos.

7) ¿Le parece una pregunta importante para que se haga en una consulta médica?

Para las respuestas

1) Lea detenidamente las opciones de respuestas.

2) ¿Hay alguna palabra de la cual no conoce el significado?

3) ¿Usaría otras palabras? Ejemplos.

4) ¿Falta alguna opción?

5) ¿Sobra alguna opción?

Para el final de la entrevista

1) ¿Cree que habría que agregar alguna pregunta? ¿Cuál/es? ¿Por qué?

2) ¿Hay algo importante que no se preguntó? ¿Qué?

3) ¿Cree que habría que quitar alguna pregunta? ¿Cuál/es? ¿Por qué?

4) ¿Le parece adecuado que se hagan estas preguntas en la consulta médica aunque el motivo de consulta sea otro?

5) ¿Por qué?

concordancia entre la información obtenida por dos entrevistadores diferentes en una misma entrevistada), su estabilidad (la concordancia entre las respuestas expresadas por una misma persona en dos momentos distintos con iguales condiciones) y su coherencia interna (la fuerza de la correlación entre las preguntas que exploran la violencia física/ psicológica y la violencia sexual/psicológica). La reproducibilidad se evaluó en el grupo $\mathrm{AB}$, mientras que la estabilidad se estudió en el grupo $C D$, en ambos casos mediante el nivel de concordancia kappa para cada pregunta. Teniendo en cuenta que cada dominio se exploró mediante una sola pregunta, para la coherencia interna se aceptó un coeficiente de correlación alfa entre 0,4 y 0,7.

Debido a que no existe un método estándar para la detección de la VBG, se comparó la frecuencia de casos de VBG detectados en los grupos AB y $\mathrm{CD}$ con la frecuencia de situaciones de VBG detectadas en el grupo de ayuda compuesto por víctimas de la violencia que son atendidas en el PMIG.

Los profesionales de la salud que participaron en la presente investigación estaban capacitados para detectar situaciones de violencia hacia la mujer y para prestar la debida asistencia en el ámbito de la atención primaria. Las mujeres que participaron lo hicieron de forma voluntaria y anónima y firmaron una declaración de consentimiento informado. Este proyecto contó con la aprobación del Comité de Ética del Hospital de Clínicas José de San Martín, de la Universidad de Buenos Aires (UBA), Argentina.

\section{RESULTADOS}

Como resultado de la validación realizada se introdujeron cambios en los enunciados de las preguntas y se comprobó que exploraban correctamente las distintas manifestaciones de violencia y que cada una de ellas contaba con una adecuada gama de ejemplos. Las opciones de respuesta ("Sí", "No", y "No desea contestar") resultaron adecuadas, tanto para la detección de situaciones de violencia pasadas como actuales. Sin embargo, la pregunta dirigida a valorar la posibilidad de sufrir actos de VBG en el momento de la entrevista debió modificarse, ya que las encuestadas en un trabajo previo la relacionaron con la inseguridad en la vía pública (5).

En cuanto a la validación lingüística, el análisis cualitativo de las entrevistas demostró que las mujeres encuestadas comprendieron bien las preguntas, los ejemplos y las opciones de respuesta y no mostraron dificultades para comprender el léxico empleado, por lo que no fue necesario realizar cambios sustanciales. La amplia variedad de ejemplos para situaciones de violencia psíquica y física se consideró muy satisfactoria, especialmente la mención de formas "leves" de agresión que permitió "legitimar" la percepción de las mismas como formas de violencia. Un aporte importante de este trabajo es haber introducido el maltrato a los hijos como una forma de maltrato emocional a la mujer.

Las mujeres encuestadas no percibieron el cuestionario aplicado como un elemento intimidatorio ni una intromisión en su vida privada y consideraron su aplicación en el ámbito de la atención primaria como una oportunidad clave para expresar su sufrimiento y recibir ayuda.

Como resultado de estos procesos de validación se obtuvo un cuestionario comprendido y aceptado por las mujeres que participaron en el estudio de validación psicométrica (cuadro 2) y que puede ser aplicado por profesionales de atención primaria de salud.

El nivel de concordancia encontrado entre las respuestas del grupo $\mathrm{AB}-\mathrm{en}$ el que se evaluó la reproducibilidad- $\mathrm{y}$ entre las respuestas del grupo $C D$ - en el que se evaluó la estabilidad- fue muy alto (cuadro 3). La coherencia interna, evaluada mediante el coeficiente de correlación alfa, fue de 0,755 entre la violencia psíquica y la física, de 0,498 entre la violencia psíquica y la sexual, de 0,596 entre la violencia física y la sexual, y de 0,715 entre las diferentes manifestaciones de violencia (psíquica, física, sexual y abuso durante la niñez).

Si bien se estudió un reducido número de mujeres - ya que el objetivo de esta investigación no era estimar la prevalencia de VBG en la población-, la frecuencia de situaciones de violencia detectadas con este cuestionario en los grupos $\mathrm{AB}$ y $\mathrm{CD}$ (cuadro 4) fue similar a los valores de prevalencia encontrados en la Argentina (5), aunque, como era de esperar, resultó muy inferior a la detectada entre las mujeres 
CUADRO 2. Características demográficas de la muestra ${ }^{a}$

\begin{tabular}{lrr}
\hline \multicolumn{1}{c}{ Característica } & No. & $\%$ \\
\hline Años de educación & & \\
$\quad$ Más de 11 años & 38 & 56,7 \\
$\quad$ Entre 7 y 11 años & 22 & 32,8 \\
$\quad$ Menos de 7 años & 7 & 10,5 \\
Situación de pareja & & \\
$\quad$ Pareja conviviente & 36 & 53,7 \\
$\quad$ Pareja no conviviente & 8 & 11,9 \\
$\quad$ Sin pareja & 23 & 34,4 \\
Realiza tareas remuneradas & & \\
$\quad$ Sí & 37 & 55,2 \\
$\quad$ No & 24 & 35,8 \\
Jubilados & 6 & 9,0 \\
\hline a $N=67$; edad (mediana) 44,5 años (recorrido de 22 a 82 \\
años).
\end{tabular}

que asistían a las actividades de apoyo a víctimas de la violencia (cuadro 4).

\section{DISCUSIÓN}

Después de la validación, iniciada por la Asociación de Planificación Familiar (Plafam) de Venezuela (12) y continuada en el PMGI, este cuestionario mostró situaciones de VBG con mayor sensibilidad que las preguntas inespecíficas que se hacen habitualmente $(40 \%$ frente a $8 \%$, respectivamente) y aportó una mayor capacidad de discriminación entre las distintas modalidades de violencia. Además, sus propiedades lingüísticas y psicométricas resultaron adecuadas.

Es de suma importancia contar con un cuestionario sensible, confiable y específico que pueda aplicarse para detectar casos de VBG en la práctica médica. La generalización de su uso podría mejorar las posibilidades de comparar estudios descriptivos realizados en distintas poblaciones. A pesar de la sencillez aparente del cuestionario validado en este estudio, su aplicación debe estar en manos de profesionales capacitados para la atención de casos de violencia de género.

Explorar en el ámbito de la consulta médica las manifestaciones de VBG es en sí una intervención útil que manifiesta el reconocimiento externo de una experiencia negativa "inherente a

CUADRO 3. Valores de concordancia kappa en la evaluación de la reproducibilidad y de la estabilidad para cada pregunta

\begin{tabular}{|c|c|c|}
\hline \multirow[b]{2}{*}{ Pregunta } & \multicolumn{2}{|c|}{ Valor de kappa } \\
\hline & Reproducibilidad ${ }^{a}$ & Estabilidad $^{\mathrm{b}}$ \\
\hline Violencia psíquica & 1,00 & 0,93 \\
\hline Violencia física & 1,00 & 0,89 \\
\hline Violencia sexual & 1,00 & 1,00 \\
\hline Abuso sexual en la niñez & 1,00 & 0,91 \\
\hline Percepción de riesgo actual & 0,63 & 0,62 \\
\hline
\end{tabular}

CUADRO 4. Frecuencia de detección de situaciones de violencia basada en género (VBG) y de percepción de riesgo actual, según los grupos

\begin{tabular}{lccc}
\hline & \multicolumn{3}{c}{ Frecuencia, \% } \\
\cline { 2 - 4 } \multicolumn{1}{c}{ Modalidad de VBG } & $\operatorname{Grupo~AB}^{\mathrm{a}}(n=32)$ & Grupo CD $^{\mathrm{b}}(n=35)$ & Víctimas de VBG $(n=10)$ \\
\hline Violencia psicológica & 40,6 & 37,1 & 100 \\
Violencia física & 34,4 & 17,1 & 100 \\
Abuso sexual durante la niñez & 21,9 & 22,9 & 12,5 \\
Violencia sexual & 28,1 & 11,4 & 75,0 \\
Percepción de riesgo actual & 15,0 & 13,0 & 62,5 \\
\hline
\end{tabular}

a Grupo en el que se evaluó la reproducibilidad.

b Grupo en el que se valuó la estabilidad.

la vida privada" y para ello es necesario contar con profesionales sensibles, capacitados y entrenados en la detección de los casos y en la atención de las víctimas (13). Sin embargo, en la actualidad los médicos clínicos no están suficientemente familiarizados con este tema, como se comprobó en un estudio realizado en la Argentina (14).

Hasta el momento, la Fuerza de Tareas para la Prevención, de los Estados Unidos de América y Canadá, no ha encontrado suficientes elementos que justifiquen establecer el tamizaje de VBG en las consultas clínicas de esos países $(15,16)$, en parte debido a la falta de estudios que permitan valorar los beneficios de esa medida.

Es necesario que la VBG reciba la atención que merece en los programas de promoción, protección y cuidado de la salud, lo que conduciría a cambios en la cultura institucional en torno a este tema. El sector de la salud puede convertirse en un protagonista central en el tratamiento de las situaciones de VBG. Así, con su acción complementaria a los mecanismos legales y su asistencia espe- cializada se podrían beneficiar tanto las mujeres que buscan activamente ayuda por sufrir actos de VBG como las que de forma recurrente exigen atención médica por "padecimientos crónicos" originados por el maltrato silenciado.

Los resultados del proceso de validación realizado demostraron la solidez de este instrumento breve de evaluación, tanto por su formato y su contenido como por su comprensibilidad y confiabilidad. Este instrumento puede emplearse en las consultas clínicas para detectar situaciones de violencia psicológica, física y sexual, así como de abuso sexual durante la niñez.

Este cuestionario validado para la detección sistemática de casos de VBG en los servicios de salud puede ser un elemento importante en la estrategia para disminuir la violencia hacia la mujer en nuestra Región.

Agradecimientos. El presente proyecto de investigación se realizó en el marco de la Beca Carrillo-Oñativia, otorgada por el Ministerio de Salud de la Argentina. Los autores agradecen la 
colaboración y apoyo de Paula Alemán, Mireya Aznar, Sandra Braun, Leonardo Castresana y María de las
Mercedes Dabat, del Programa de Medicina Interna General, Hospital de Clínicas, Universidad de Buenos Aires,
Argentina, de Viviana Mazur de la Asociación de Medicina General de Buenos Aires, y de Fabián Portnoy.

\section{REFERENCIAS}

1. World Health Organization. Violence-a global public health problem. World report on violence and health. Geneva: WHO; 2002.

2. Convención Interamericana para prevenir, sancionar y erradicar la violencia contra la mujer. Washington, D.C.: Organización de Estados Americanos; 1994. Hallado en http:/ / www.oas.org/juridico/spanish/tratados/ a-61.html. Acceso el 12 de enero de 2005.

3. Coker A, Smith P, Bethea L, King MR, McKeown RE. Physical health consequences of physical and psychological intimate partner violence. Arch Fam Med. 2000;9:451-7.

4. McCauley J, Kern D, Kolodner K, Dill L, Schroeder A, DeChant H, et al. The "battering syndrome": prevalence and clinical characteristics of domestic violence in primary care internal medicine practices. Ann Intern Med. 1995;123:737-46.

5. Pontecorvo C, Mejía R, Alemán M, Vidal A, Majdalani MP, Fayanás R, et al. Violencia doméstica contra la mujer. Una encuesta en consultorios de atención primaria. Medicina (Buenos Aires). 2004;64:482-96.

6. International Planned Parenthood Federation (IPPF). Estrategias para el tratamiento y la prevención de los efectos de la violencia doméstica en la salud mental. Chicago: American Medical Association; 1995.

7. International Planned Parenthood Federation (IPPF). Estrategias para el tratamiento y la prevención de la violencia sexual. Chicago: American Medical Association; 1995.

8. Smith PH, Earp JA, de Vellis R. Measuring battering: development of the Women's Experience with Battering (WEB) scale. Womens Health. 1995;(4):273-88.

9. McFarlane J, Parker B, Soeken K, Bullock L. Assessing for abuse during pregnancy. Severity and frequency of injuries and associated entry into prenatal care. JAMA. 1992;267: 3176-8.

10. Straus M, Hamby S, Mc Coy S, Sugarman D. The revised conflict tactics scales (CTS2). J Fam Issues. 1996;17:283-316.

11. Feldhaus K, McLain JK, Holly L. Accuracy of three brief screening questions for detecting partner violence in the emergency department. JAMA. 1997;277:1357-61.

12. International Planned Parenthood Federation Detección sistemática de violencia basada en género. Boletín sobre cómo integrar la violencia basada en género en la salud sexual y reproductiva. ¡BASTA! 2000;6. Hallado en: http://www.ippfwhr.org/publications/down load/serial_issues/basta2000summer_s.pdf

13. Liebschutz J, Frayne S, Saxe G. Violence against women. Boston: ACP Women's Health; 2003.

14. Mejía R., Alemán M, Fernández A, Pérez Stable EJ. Conocimiento y detección de violencia doméstica por los médicos clínicos. Medicina (Buenos Aires). 2000;60:591-4.

15. U.S. Preventive Services Task Force. Screening: family an intimate partner violence. Rockville, Maryland: Agency for Healthcare Research and Quality; 2004. Hallado en: www.ahrq.gov/clinic/uspstfix.htm. Acceso el 12 de enero de 2005.

16. Canadian Task Force on Preventive Health Care. Prevention and treatment of violence against women: systematic review and recommendations. NGC summary. Rockville, Maryland: National Guideline Clearinghouse; 2004. Hallado en: http://www.guideline. gov/summary/summary.aspx?doc_id=3657 \&nbr=2883\&string=violence Acceso el 12 de enero 2005.

Manuscrito recibido el 30 de septiembre de 2004. Aceptado para publicación, tras revisión, el 11 de noviembre de 2004.
ABSTRACT

\section{Validation of a short questionnaire to use in clinical consultations to detect gender violence}

Objective. To validate a Spanish-language questionnaire to be used in primary care consultations to detect cases of violence against women.

Methods. The validation study was carried out between October 2002 and October 2003 in the Program of General Internal Medicine of the Clinical Hospital of the University of Buenos Aires, in the city of Buenos Aires, Argentina. The original version of this Spanish-language questionnaire had been developed in sexual and reproductive health clinics of five countries of Latin America and the Caribbean. The questionnaire covered the most frequent forms of gender-based violence (emotional, physical, sexual, and sexual abuse during childhood); explored how and when the violence occurred and who perpetrated it; and investigated the patient's perception of her current risk. In the research in Buenos Aires the format and theoretical content of the survey were evaluated, and linguistic and psychometric validations were also carried out. The reliability of the tool was assessed in terms of its reproducibility, stability, and internal consistency.

Results. The study found that the women surveyed in Buenos Aires had a good understanding of the questions, the examples given with the questions, and the response options. The women did not perceive the survey as being intimidating or as an intrusion into their private lives. The women generally regarded the use of the survey in primary care settings as a key opportunity for them to express their suffering and to receive assistance. The level of agreement for the answers to the questions in all five areas (psychological violence, physical violence, sexual violence, sexual abuse during childhood, and perception of current risk) was very high, in terms of both reproducibility (kappa values of 0.63 to 1.00 ) and stability (kappa values of 0.62 to 1.00). The alpha correlation coefficient for internal consistency was 0.755 for psychological violence and physical violence, 0.498 for psychological violence and sexual violence, 0.596 for physical violence and sexual violence, and 0.715 for the different manifestations of violence (psychological, physical, sexual, and abuse during childhood).

Conclusion. This research demonstrated the soundness of the brief questionnaire, in terms of its format, content, comprehensiveness, and reliability. This instrument can be used in clinical consultations to detect situations of psychological violence, physical violence, sexual violence, and sexual abuse during childhood.

Keywords Domestic violence, validation studies, Argentina. 